IPMU 08-0015

\title{
Non-Gaussianity from Symmetry
}

\author{
Teruaki Suyama ${ }^{1}$ and Fuminobu Takahashi ${ }^{2}$ \\ ${ }^{1}$ Institute for Cosmic Ray Research, \\ University of Tokyo, Kashiwa 277 8582, Japan, \\ 2 Institute for the Physics and Mathematics of the Universe, \\ University of Tokyo, Chiba 277-8568, Japan
}

\begin{abstract}
We point out that a light scalar field fluctuating around a symmetry-enhaced point can generate large non-Gaussianity in density fluctuations. We name such a particle as an "ungaussiton", a scalar field dominantly produced by the quantum fluctuations, generating sizable non-Gaussianity in the density fluctuations. We derive a consistency relation between the bispectrum and the trispectrum, $\tau_{\mathrm{NL}} \sim 10^{3} \cdot f_{\mathrm{NL}}^{4 / 3}$, which can be extended to arbitrary high order correlation functions. If such a relation is confirmed by future observations, it will strongly support this mechanism.
\end{abstract}




\section{INTRODUCTION}

The concept of symmetry has been a guiding principle in modern physics. The structure of the standard model $(\mathrm{SM})$ is dictated by the SM gauge symmetries, $S U(3)_{C} \times S U(2)_{L} \times$ $U(1)_{Y}$. In the string theory, gauge symmetries as well as discrete global symmetries are ubiquitous [1]].

One of the plausible candidates for the theory beyond SM is supersymmetry (SUSY), which relates a particle to a superparticle with different statistics. In particular, a superpartner of a fermion field with spin $1 / 2$ is a scalar field. Therefore there are perhaps many fundamental (or composite) scalar fields in nature, and most of them may be charged under some symmetries.

A scalar field that is singlet under any symmetries does not possess a special point in its field space, and so, the minimum of the potential may vary in the evolution of the universe. The change of the minimum generically induces coherent oscillations of the scalar field. If the scalar field has only interactions suppressed by the Planck scale, $M_{P}\left(=2.4 \times 10^{18} \mathrm{GeV}\right)$, it might induce notorious cosmological moduli problem [2], which has recently turned out to be more acute than previously thought [3, 4, 5]. That is to say, the modulus decay generically produces too many gravitinos, which either spoil the success of the big bang nucleosynthesis or exceed the observed dark matter abundance. The moduli problem and the moduli-induced gravitino problem can be ameliorated if the initial displacement is somehow suppressed. This, however, is difficult to achieve unless the modulus field is charged under some symmetries.

On the other hand, a scalar field charged under some symmetries has a special point in its field space ${ }^{a}$, where the symmetries are enhanced and the scalar potential has its extremum. If the symmetry-enhanced point is the (local) minimum of the potential, it is quite likely that the scalar field sits at the point from the beginning of the universe and continues to sit there [6, 7, 8, 9, 10]. Such a scalar field apparently does not affect the

\footnotetext{
a One exception is an axion with a shift symmetry, because the axion does not have a symmetry-enhanced point. We will make a comment on this case later.
} 
subsequent evolution of the universe, since its energy density vanishes, at least classically. At the quantum level, however, this may not hold as we describe below.

The inflation is now strongly suggested by the recent observations of the cosmic microwave background (CMB). During inflation, the inflaton acquires quantum fluctuations, which become the seed for the present structures of the universe. Interestingly, any scalar fields of masses smaller than the expansion rate during inflation, $H_{I}$, similarly acquire quantum fluctuations of $O\left(H_{I}\right)[11]$.

We point out that such light scalar fields fluctuating around the symmetry-enhanced point necessarily induce non-Gaussianity at some level in the observed CMB power spectrum ${ }^{b}$. In other words, the scalar fields whose energy classically vanishes must have left their traces in the CMB; those scalar fields are produced by the purely quantum fluctuations, making the fluctuations in their energy density deviate from the Gaussian distribution. We call such a scalar particle an "ungaussiton", because their energy density exhibits strong non-Gaussianity [12, 13]. Our definition of the ungaussiton is an elementary or composite scalar field dominantly produced by the quantum fluctuations, generating sizable non-Gaussianity in the density fluctuations. Note that the definition itself is not related to the symmetry. The presence of the symmetry is one of the ways to naturally make a scalar field to be an ungaussiton.

If there are indeed many scalar fields with symmetries in nature, some of them may be light during inflation. Then they can be ungaussitons. In this way we expect that the presence of non-Gaussianity might be quite generic in our vacuum. Interestingly, it has been recently reported that large non-Gaussianity was detected by the analysis on the WMAP 3yr data [15]. The latest WMAP 5yr data seem to have the same tendency, although the vanishing non-Gaussianity is allowed within 95\% C.L. [16]. Those hints on the non-Gaussianity, or its future detection, may be originated from such particles, i.e., ungaussitons.

\footnotetext{
${ }^{\mathrm{b}}$ As will be discussed later, the symmetries are not necessarily exact. Our arguments remain intact as long as the deviation from the potential minimum is small (or comparable) relative to the Hubble parameter during inflation.
} 
In this paper we study the ungaussiton mechanism and write down a condition for the non-Gaussianity to become large enough to be observed by the ongoing and planned observation such as the WMAP and Planck satellites. Moreover, we derive a consistency relation between the bispectrum and the trispectrum, which, if confirmed, will be a smoking gun of the ungaussiton mechanism. Importantly, the bispectrum and the trispectrum predicted in the ungaussiton scenario exhibit a specific dependence on the scale; both get enhanced as one goes to smaller scales. Such a feature on the scale dependence as well as the consistency relation between the bispectrum and the trispectrum will help us to distinguish our scenario from the others.

Let us here comment on how the ungaussiton can remain around the minimum of the potential during inflation. As is well known, if inflation lasts sufficiently long, a light scalar field (an ungaussiton in our case) relaxes towards the Bunch-Davies (BD) distribution [14]. The BD distribution is such that the root mean square (RMS) of the field fluctuations on large scales is given by $\mathcal{O}\left(H_{I}^{2} / m\right)$, where $m$ is the effective mass of the ungaussiton during inflation. If $m$ is much smaller than $H_{I}$, the BD distribution tells us that the ungaussiton takes a value of $\mathcal{O}\left(H_{I}^{2} / m\right) \gg H_{I}$ in most regions, while there is a small portion of the whole universe where the field value is of $\mathcal{O}\left(H_{I}\right)$. If our observable universe happens to be inside such small region, the spatial average of the ungaussiton over the observable universe can be very close to the origin. This may sound a fine-tuning, but it may not. There may be anthropic arguments to select such regions, although there is no justification for such reasoning at this moment. There are other ways to keep the ungaussiton near the origin. One possibility is that the mass $m$ is not much smaller than $H_{I}$. Then the RMS values of the ungaussiton is not that large compared to $H_{I}$, which makes it easy to find such regions that the field value is of $O\left(H_{I}\right)$ without fine-tunings. Another is that the last inflation was not long enough. For instance, the $e$-foldings of $50 \sim 60$ is too short for the field to reach the $\mathrm{BD}$ distribution. The ungaussiton then stays near the minimum of the potential throughout the inflation, if the ungaussiton dynamically relaxed to the origin before the inflation. We will come back to this issue in Sec. IV. 


\section{NON-GAUSSIANITY FROM AN UNGAUSSITON}

Let us consider a scalar field, $\sigma$, which is charged under some symmetries ${ }^{\mathrm{c}}$. We set the origin of $\sigma$ to be the symmetry-enhanced point, which is assumed to be the (local) minimum of the potential. If it is a meta-stable vacuum, the life time of the vacuum is assumed to be much longer than the present age of the universe. The potential around the origin can be obtained by expanding the scalar potential in terms of $\sigma$. We assume that the potential is well approximated by the quadratic potential d, and that it is valid at least up to $\sigma=O\left(H_{I}\right)$ :

$$
U(\sigma)=\frac{1}{2} m_{\sigma}^{2} \sigma^{2}
$$

where $m_{\sigma}$ denotes the mass of $\sigma^{\mathrm{e}}$. The symmetry of $U(\sigma)$ is the $Z_{2}$ symmetry in this case. If $m_{\sigma}$ is larger than $H_{I}$, the fluctuations of $\sigma$ get suppressed, while $\sigma$ acquires fluctuations if $m_{\sigma}<H_{I}$. In the following we focus on the case of $m_{\sigma}<H_{I}$.

We first give a rough sketch of how non-Gaussianity is generated in the primordial curvature fluctuations. During inflation, the scalar field $\sigma$ likely sits at the origin, $\sigma=0$, based on the symmetry argument. For the moment we assume this is the case, and we will discuss this issue in detail in Sec. IV. Since the mass is lighter than the Hubble parameter, $\sigma$ acquires quantum fluctuations, $\delta \sigma$, around the origin. The magnitude of $\delta \sigma$ is roughly equal to $H_{I}$. After inflation, $\sigma$ starts to oscillate around the origin when the Hubble parameter becomes comparable to $m_{\sigma}$, with an amplitude taking a different value in each local patch of the universe. The energy density of $\sigma$ is proportional to the amplitude squared, $(\delta \sigma)^{2}$ f. Taking $\delta \sigma$ as a Gaussian variable, the resultant energy density exhibits strong deviation from the Gaussian distribution [12, 17], hence the name

\footnotetext{
c It is easy to extend our results to a case of the multiple scalars.

d One can also consider a case that a quartic potential dominates over the quadratic one. The resultant non-Gaussianity tends to be smaller in this case.

e As mentioned before, the effective mass during inflation may be different from that in the low energy. Our arguments remains intact as long as the effective mass is smaller than the Hubble parameter.

f Precisely speaking, one has to take account of the spatially averaged value of $\sigma$, which does not vanish in the observable finite universe. Then the dependence on $\delta \sigma$ becomes slightly modified. Nevertheless this intuitive explanation gives a qualitatively valid picture.
} 
an ungaussiton. For simplicity, we assume that the ungaussiton $\sigma$ decays into radiation in the visible sector ${ }^{g}$. Then, the fluctuations in the energy density of the ungaussiton turn into small corrections to the adiabatic fluctuations arising from the inflaton. Although the corrections might be small in the amplitude of the curvature perturbations, they can significantly change higher order correlation funcitions such as the three-point function which characterizes the strength of non-Gaussianty.

Let us now estimate non-Gaussianity produced by the ungaussiton. First we define the fluctuations of $\sigma$, taking account of the fact that our observable universe is finite. Assume that an ensemble average of $\sigma$ vanishes. Since our observable universe is finite, the spatial average of $\sigma$ over the comoving volume corresponding to the present Hubble horizon does not vanish. In order to calculate the spatial average of $\sigma$, we need to define the time slicing. For later convenience, we take a flat time slicing at $t=t_{*}$ when the smallest scale of interest leaves the horizon during inflation. Let us denote the spatial average of $\sigma$ on the flat slicing by $\bar{\sigma}$ and define the fluctuation $\delta \sigma_{*}(\vec{x})$ as $\delta \sigma_{*}(\vec{x}) \equiv \sigma\left(t_{*}, \vec{x}\right)-\bar{\sigma}$. If the mass of $\sigma$ is much lighter than $H_{I}$, those fluctuations defined on the slicing can be evaluated when each scale leaves the horizon. In the following we assume that the inflation lasts long enough that $\sigma$ acquires an almost scale-invariant Gaussian fluctuations which extend beyond the present Hubble horizon. To put it more precisely, we define the fluctuations in a box of a size $L$, which is (at least) several times larger than the present Hubble horizon. In fact, the fluctuations of the scales beyond the current horizon size contribute to the non-vanishing spatial average of $\sigma$.

According to the $\delta N$ formalism [19, 20, 21, 22], on sufficiently large scales, the curvature perturbation $\zeta$ on the uniform energy density hypersurface at $t=t_{f}$ is equal to the perturbations in the number of e-foldings between the uniform density slicing at $t=t_{f}$

$\mathrm{g}$ The ungaussiton can generate sizable non-Gaussianity even if it does not decay. In this case, the non-Gaussianity arises from the isocurvature fluctuations, which leave the characteristic signatures in the CMB anisotropy [18]. Indeed, the $Z_{2}$ symmetry of the ungaussiton may naturally account for the stability to become dark matter. 
and the initial flat slicing at $t=t_{*}$ :

$$
\zeta\left(t_{f}, \vec{x}\right)=N\left(t_{f}, t_{*}, \vec{x}\right)-\bar{N}\left(t_{f}, t_{*}\right)
$$

with

$$
\begin{aligned}
N\left(t_{f}, t_{*}, \vec{x}\right) & =\int_{t_{*}}^{t_{f}} H(t, \vec{x}) d t, \\
\bar{N}\left(t_{f}, t_{*}\right) & =\int_{t_{*}}^{t_{f}} \bar{H}(t) d t,
\end{aligned}
$$

where $N\left(t_{f}, t_{*}, \vec{x}\right)$ is the number of e-foldings of expansion between the two slicings, and $\bar{N}$ is that of the background universe (or more precisely, the spatially averaged one). Similarly, $H(t, \vec{x})$ denotes the local expansion rate, while $\bar{H}(t)$ denotes the background one.

We assume that the ungaussiton $\sigma$ decays after the reheating ${ }^{\mathrm{h}}$, and set $t_{f}$ at the time well after the decay of $\sigma$. As mentioned, we take $t_{*}$ at the time when the smallest scale of interest crossed the Hubble horizon during inflation. The curvature perturbation $\zeta$ is conserved after $t=t_{f}$, that is, $\zeta$ becomes independent of $t_{f}$, since the adiabatic pressure condition is satisfied. Then we can regard the number of the $e$-foldings as the function of $\phi_{*} \equiv \phi\left(t_{*}, \vec{x}\right)$ and $\sigma_{*} \equiv \sigma\left(t_{*}, \vec{x}\right)$, and expand $\zeta$ in terms of the fluctuations $\delta \phi_{*}$ and $\delta \sigma_{*}$, which are the perturbations of $\phi_{*}$ and $\sigma_{*}$, as

$$
\zeta \approx N_{\phi} \delta \phi_{*}+N_{\sigma} \delta \sigma_{*}+\frac{1}{2} N_{\sigma \sigma} \delta \sigma_{*}^{2}+\frac{1}{6} N_{\sigma \sigma \sigma} \delta \sigma_{*}^{3}+\frac{1}{24} N_{\sigma \sigma \sigma \sigma} \delta \sigma_{*}^{4}+\cdots,
$$

where the indices denote the partial derivative with respect to the variables, i.e., $N_{\phi}=$ $\partial N / \partial \phi, N_{\sigma}=\partial N / \partial \sigma$, etc.. Those coefficients depend on the background evolution of the scalar fields $\phi$ and $\sigma$ from $t=t_{*}$ until $t=t_{f}$. In particular, we use the spatially averaged value, $\sigma=\bar{\sigma}$, as the initial condition of the background evolution. Here and in what follows we omit the dependence on the comoving coordinate $\vec{x}$ unless otherwise stated. We assume that the first term on the right hand side (RHS) of Eq. (4), coming from the inflaton

$\mathrm{h}$ This is just for the sake of simplicity. If it is the inflaton that decays later, we should take $t_{f}$ after the reheating. However, the non-Gaussianity tends to be suppressed in this case, since the energy density of the ungaussiton becomes small. 
fluctuations, dominates the two-point function of $\zeta$. As for the relevant higher order correlation functions, we assume that the leading contributions come from the ungaussiton fluctuations, and we have neglected second and higher order terms in $\delta \phi_{*}$ in Eq. (4). It is known that the non-Gaussianity of $\zeta$ coming from the intrinsic non-Gaussianity of $\delta \phi_{*}$ and $\delta \sigma_{*}$ is far below the observational sensitivity such as Planck [23, 24, 25, 26]. Hence we can treat both $\delta \phi_{*}$ and $\delta \sigma_{*}$ as the Gaussian variables ${ }^{\mathrm{i}}$.

Before going further, it will be worth mentioning how the symmetry comes into the game. The expression (4) itself is rather generic, but, what is peculiar to the ungaussiton mechanism is the relative size of the coefficients. To see this, let us concentrate on $N_{\sigma}$, which depends on the background evolution of $\sigma$. As mentioned above, we take $\sigma=\bar{\sigma}$ as the initial condition. Let us expand $N_{\sigma}$ around another initial condition, $\sigma=0$.

$$
\left.N_{\sigma}\right|_{\bar{\sigma}}=\left.N_{\sigma}\right|_{0}+\left.N_{\sigma \sigma}\right|_{0} \bar{\sigma}+\left.\frac{1}{2} N_{\sigma \sigma \sigma}\right|_{0} \bar{\sigma}^{2}+\cdots
$$

where we have explicitly shown the initial values of $\sigma$. What the $Z_{2}$ symmetry tells us is that the potential, and therefore the background evolution, is even around the origin. That is to say, the functions $N_{\sigma}$ and $N_{\sigma \sigma \sigma}$ should vanish when $\sigma=0$ is used as the initial condition. That is,

$$
\left.N_{\sigma}\right|_{0}=0,\left.\quad N_{\sigma \sigma \sigma}\right|_{0}=0
$$

Therefore the expansion of $\left.N_{\sigma}\right|_{\bar{\sigma}}$ around $\sigma=0$ actually starts with a term linear in $\bar{\sigma}$. Noting that $\bar{\sigma}$ is actually comparable to the fluctuations $\delta \sigma_{*}$, one can see that the term linear in $\delta \sigma_{*}$ in (44) is comparable to the term quadratic in $\delta \sigma_{*}$. Intuitively speaking, since $\sigma$ stays at the origin based on the symmetry arguments, if one expands a function of $\sigma$, $f(\sigma)$, around the origin, the leading term should start with $\sigma^{2}$, as long as $f(\sigma)$ satisfies $f(\sigma)=f(-\sigma)$ in the interested range of $\sigma$. When one takes account of the finiteness of the current horizon scale, this feature appears in the suppression of $\left.N_{\sigma}\right|_{\bar{\sigma}}$ as shown above. Let us emphasize again that the suppression is due to the presence of the symmetry, and that

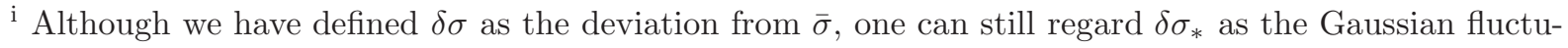
ations. This is because the superhorizon fluctuations contribute to $\bar{\sigma}$, while $\delta \sigma$ represents fluctuations inside the horizon.
} 
this makes the higher order term quadratic in $\delta \sigma_{*}$ very important. For the same reason, the cubic term of $\delta \sigma_{*}$ is comparable to the quartic one, and therefore we keep the quartic term of $\delta \sigma_{*}$ for the moment.

The power spectrum $P_{\zeta}$, bispectrum $B_{\zeta}$ and trispectrum $T_{\zeta}$ of the curvature perturbations are defined as

$$
\begin{aligned}
& \left\langle\zeta_{\vec{k}_{1}} \zeta_{\vec{k}_{2}}\right\rangle_{c}=(2 \pi)^{3} \delta\left(\vec{k}_{1}+\vec{k}_{2}\right) P_{\zeta}\left(k_{1}\right), \\
& \left\langle\zeta_{\vec{k}_{1}} \zeta_{\vec{k}_{2}} \zeta_{\vec{k}_{3}}\right\rangle_{c}=(2 \pi)^{3} \delta\left(\vec{k}_{1}+\vec{k}_{2}+\vec{k}_{3}\right) B_{\zeta}\left(k_{1}, k_{2}, k_{3}\right), \\
& \left\langle\zeta_{\vec{k}_{1}} \zeta_{\vec{k}_{2}} \zeta_{\vec{k}_{3}} \zeta_{\vec{k}_{4}}\right\rangle_{c}=(2 \pi)^{3} \delta\left(\vec{k}_{1}+\vec{k}_{2}+\vec{k}_{3}+\vec{k}_{4}\right) T_{\zeta}\left(\vec{k}_{1}, \vec{k}_{2}, \vec{k}_{3}, \vec{k}_{4}\right),
\end{aligned}
$$

where $\zeta_{\vec{k}}$ are Fourier components of $\zeta$, i.e., $\zeta_{\vec{k}} \equiv \int d^{3} x e^{-i \vec{k} \cdot \vec{x}} \zeta(\vec{x}), k_{i} \equiv\left|\vec{k}_{i}\right|$ for $i=1 \cdots 4$, and the subscript $c$ means that we take the connected part of the corresponding correlator. It is useful to define $\mathcal{P}_{\zeta} \equiv k^{3} /\left(2 \pi^{2}\right) P_{\zeta}$. Let us also define the power spectra of $\delta \phi$ and $\delta \sigma$,

$$
\begin{aligned}
\left\langle\delta \phi_{\vec{k}_{1}} \delta \phi_{\vec{k}_{2}}\right\rangle & =(2 \pi)^{3} \delta\left(\vec{k}_{1}+\vec{k}_{2}\right) P_{\phi}\left(k_{1}\right), \\
\left\langle\delta \sigma_{\vec{k}_{1}} \delta \sigma_{\vec{k}_{2}}\right\rangle & =(2 \pi)^{3} \delta\left(\vec{k}_{1}+\vec{k}_{2}\right) P_{\sigma}\left(k_{1}\right), \\
\mathcal{P}_{\phi(\sigma)} & \equiv \frac{k^{3}}{2 \pi^{2}} P_{\phi(\sigma)} .
\end{aligned}
$$

Neglecting the tilt of the power spectra, we have

$$
\mathcal{P}_{\phi} \simeq \mathcal{P}_{\sigma} \approx\left(\frac{H_{I}}{2 \pi}\right)^{2}
$$

As mentioned above, the inflaton dominates the two-point correlator of $\zeta$, i.e.,

$$
P_{\zeta} \simeq N_{\phi}^{2} P_{\phi}
$$

As for the bispectrum, the dominant contributions come from the ungaussiton, which, up to sixth order in $\delta \sigma_{*}$, gives

$$
\begin{aligned}
B_{\zeta}\left(k_{1}, k_{2}, k_{3}\right) \simeq & \frac{N_{\sigma}^{2} N_{\sigma \sigma}}{N_{\phi}^{4}}\left(P_{\zeta}\left(k_{1}\right) P_{\zeta}\left(k_{2}\right)+2 \text { perms. }\right) \\
& +\frac{N_{\sigma \sigma}^{3}}{N_{\phi}^{6}} \int \frac{d^{3} \vec{q}}{(2 \pi)^{3}} P_{\zeta}(q) P_{\zeta}\left(\left|\vec{k}_{1}+\vec{q}\right|\right) P_{\zeta}\left(\left|\vec{k}_{2}-\vec{q}\right|\right) \\
& +\frac{N_{\sigma} N_{\sigma \sigma} N_{\sigma \sigma \sigma}}{2 N_{\phi}^{6}}\left(\int \frac{d^{3} \vec{q}}{(2 \pi)^{3}} P_{\zeta}(q) P_{\zeta}\left(\left|\vec{k}_{2}-\vec{q}\right|\right) P_{\zeta}\left(k_{1}\right)+5 \text { perms. }\right),
\end{aligned}
$$


where the third term was not taken into account in Refs. [17, 28, 29, 30]. The first term is quartic order in $\delta \sigma_{*}$, while the remaining terms containing the momentum integral are sixth order in $\delta \sigma_{*}$.

In a similar way, the trispectrum up to eighth order in $\delta \sigma_{*}$ can be written as

$$
\begin{aligned}
T_{\zeta}\left(\vec{k}_{1}, \vec{k}_{2}, \vec{k}_{3}, \vec{k}_{4}\right) \simeq & \frac{N_{\sigma}^{2} N_{\sigma \sigma}^{2}}{N_{\phi}^{6}}\left(P_{\zeta}\left(k_{1}\right) P_{\zeta}\left(k_{2}\right) P_{\zeta}\left(k_{13}\right)+11 \text { perms. }\right) \\
& +\frac{N_{\sigma}^{3} N_{\sigma \sigma \sigma}}{N_{\phi}^{6}}\left(P_{\zeta}\left(k_{1}\right) P_{\zeta}\left(k_{2}\right) P_{\zeta}\left(k_{3}\right)+3 \text { perms. }\right) \\
& +\frac{N_{\sigma \sigma}^{4}}{N_{\phi}^{8}}\left(\int \frac{d^{3} \vec{q}}{(2 \pi)^{3}} P_{\zeta}(q) P_{\zeta}\left(\left|\vec{k}_{1}-\vec{q}\right|\right) P_{\zeta}\left(\left|\vec{k}_{2}+\vec{q}\right|\right) P_{\zeta}\left(\left|\vec{k}_{1}+\vec{k}_{3}-\vec{q}\right|\right)+2 \text { perms. }\right) \\
& +\frac{N_{\sigma} N_{\sigma \sigma}^{2} N_{\sigma \sigma \sigma}}{N_{\phi}^{8}}\left(\int \frac{d^{3} \vec{q}}{(2 \pi)^{3}} P_{\zeta}(q) P_{\zeta}\left(\left|\vec{k}_{2}+\vec{q}\right|\right) P_{\zeta}\left(\left|\vec{k}_{1}+\vec{k}_{4}-\vec{q}\right|\right) P_{\zeta}\left(k_{1}\right)+11 \text { perms. }\right) \\
& +\frac{N_{\sigma} N_{\sigma \sigma}^{2} N_{\sigma \sigma \sigma}}{N_{\phi}^{8}}\left(\int \frac{d^{3} \vec{q}}{(2 \pi)^{3}} P_{\zeta}(q) P_{\zeta}\left(\left|\vec{k}_{3}+\vec{q}\right|\right) P_{\zeta}\left(k_{1}\right) P_{\zeta}\left(k_{12}\right)+11 \text { perms. }\right) \\
& +\frac{N_{\sigma}^{2} N_{\sigma \sigma \sigma}^{2}}{2 N_{\phi}^{8}}\left(\int \frac{d^{3} \vec{q}}{(2 \pi)^{3}} P_{\zeta}(q) P_{\zeta}\left(\left|\vec{k}_{1}+\vec{k}_{3}+\vec{q}\right|\right) P_{\zeta}\left(k_{1}\right) P_{\zeta}\left(k_{2}\right)+11 \text { perms. }\right) \\
& +\frac{N_{\sigma}^{2} N_{\sigma \sigma} N_{\sigma \sigma \sigma \sigma}}{2 N_{\phi}^{8}}\left(\int \frac{d^{3} \vec{q}}{(2 \pi)^{3}} P_{\zeta}(q) P_{\zeta}\left(\left|\vec{k}_{3}-\vec{q}\right|\right) P_{\zeta}\left(k_{1}\right) P_{\zeta}\left(k_{2}\right)+11 \text { perms. }\right),
\end{aligned}
$$

where $k_{i j} \equiv\left|\vec{k}_{i}+\vec{k}_{j}\right|$. We have here dropped small logarithmic corrections proportional to $\log (L / R)$, where $R$ denotes a smoothing scale. The first three terms agree with those in [17, 29, 30].

It is conventional to express $B_{\zeta}$ and $T_{\zeta}$ in terms of the non-linearity parameters $f_{\mathrm{NL}}, \tau_{\mathrm{NL}}$ and $g_{\mathrm{NL}}$ defined by [27]

$$
\begin{aligned}
B_{\zeta}\left(k_{1}, k_{2}, k_{3}\right)=\frac{6}{5} & f_{\mathrm{NL}}\left(P_{\zeta}\left(k_{1}\right) P_{\zeta}\left(k_{2}\right)+2 \text { perms. }\right), \\
T_{\zeta}\left(\vec{k}_{1}, \vec{k}_{2}, \vec{k}_{3}, \vec{k}_{4}\right)= & \tau_{\mathrm{NL}}\left(P_{\zeta}\left(k_{13}\right) P_{\zeta}\left(k_{3}\right) P_{\zeta}\left(k_{4}\right)+11 \text { perms. }\right) \\
& +\frac{54}{25} g_{\mathrm{NL}}\left(P_{\zeta}\left(k_{1}\right) P_{\zeta}\left(k_{2}\right) P_{\zeta}\left(k_{3}\right)+3 \text { perms. }\right) .
\end{aligned}
$$

If we include the terms containing the momentum integral in $B_{\zeta}$ and $T_{\zeta}$, the parametrization of Eqs. (17) and (18) does not correctly represent the bispectrum and the trispectrum. However, if we consider some limiting configurations of the wavenumber vectors, so-called "squeezed" configurations, we can approximately express $B_{\zeta}$ and $T_{\zeta}$ in terms of $f_{\mathrm{NL}}, \tau_{\mathrm{NL}}$ and $g_{\mathrm{NL}}$. 
Let us here make another approximation which drastically simplifies our arguments. In the ungaussiton scenario, it turns out that those terms containing $N_{\sigma \sigma \sigma}$ or $N_{\sigma \sigma \sigma \sigma}$ in $T_{\zeta}$ are suppressed compared to the terms containing only $N_{\sigma}$ and $N_{\sigma \sigma}$, by the ratio of the ungaussiton energy density to the total one at the time of its decay. The ratio must be smaller than $\sim 10^{-5}$ not to exceed the primordial fluctuations produced from the inflaton. Hence if some quantity of interest starts from terms which contain only $N_{\sigma}$ and/or $N_{\sigma \sigma}$, we will drop higher order terms including $N_{\sigma \sigma \sigma}$ or $N_{\sigma \sigma \sigma \sigma}$.

In the case of the bispectrum, the squeezed configuration is such that one of $\left\{k_{i}\right\}$ with $i=1,2,3$ is much smaller than the length of the other two wavevectors; e.g., $k_{1} \ll k_{2}, k_{3}$. For such configuration, $f_{\mathrm{NL}}$ can be written as [17, 28, 29, 30]

$$
\frac{6}{5} f_{\mathrm{NL}} \simeq \frac{1}{N_{\phi}^{4}}\left(N_{\sigma}^{2} N_{\sigma \sigma}+N_{\sigma \sigma}^{3} \mathcal{P}_{\sigma} \log \left(k_{b} L\right)\right)
$$

where $k_{b} \equiv \min \left\{k_{i}\right\}(i=1,2,3)$ and $L$ is the size of a box in which the perturbations are defined. Since we are interested in the perturbation in the observable universe, $L$ should be taken to be $\mathcal{O}\left(1 / H_{0}\right)$, where $H_{0}$ is the present value of the Hubble parameter.

As for the trispectrum, the situation becomes a littile bit more complicated. If the four wavenumber vectors are such that $\min \left\{k_{i},\left|\vec{k}_{j}+\vec{k}_{\ell}\right|\right\}$ is much smaller than the other elements of $\left\{k_{i},\left|\vec{k}_{j}+\vec{k}_{\ell}\right|\right\}$, where $(i, j, \ell)=\{1,2,3,4\}, \tau_{\text {NL }}$ can be expressed by [17, 29, 30]

$$
\tau_{\mathrm{NL}} \simeq \frac{1}{N_{\phi}^{6}}\left(N_{\sigma}^{2} N_{\sigma \sigma}^{2}+N_{\sigma \sigma}^{4} \mathcal{P}_{\sigma} \log \left(k_{t} L\right)\right)
$$

where $k_{t} \equiv \min \left\{k_{i},\left|\vec{k}_{j}+\vec{k}_{\ell}\right|\right\}$. Meanwhile, if the configuration of the four wavenumber vectors is such that two of them is much smaller than the other two, $g_{\mathrm{NL}}$ is given by

$$
\frac{54}{25} g_{\mathrm{NL}} \simeq \frac{1}{N_{\phi}^{6}}\left(N_{\sigma}^{3} N_{\sigma \sigma \sigma}+3 N_{\sigma}^{2} N_{\sigma \sigma} N_{\sigma \sigma \sigma \sigma} \mathcal{P}_{\sigma} \log \left(k_{g 1} L\right)+3 N_{\sigma} N_{\sigma \sigma}^{2} N_{\sigma \sigma \sigma} \mathcal{P}_{\sigma} \log \left(k_{g 2} L\right)\right),
$$

where $k_{g 1}^{3} \equiv \min \left\{k_{i} k_{j} k_{\ell}\right\}$ and $k_{g 2}^{3} \equiv \min \left\{k_{i}^{2} k_{j}\right\}$. Note that $g_{\mathrm{NL}}$ starts from the terms linear in $N_{\sigma \sigma \sigma}$ or $N_{\sigma \sigma \sigma \sigma}$. Hence the trispectrum is dominated by $\tau_{\mathrm{NL}}$ terms if the leading non-Gaussianity is generated by the ungaussiton. Here and in what follows we neglect $g_{\mathrm{NL}}$ terms and the tilt of the power spectra, for simplicity.

The non-linearity parameters depend on the thermal history of the universe after inflation, since the coefficients such as $N_{\sigma}$ and $N_{\sigma \sigma}$ depend on the evolution of the inflaton 
and the ungaussiton. We consider a case that the ungaussiton decays after the reheating, since we are interested in the case that the non-Gaussianity becomes large. That is to say, we focus on the case of

$$
\Gamma_{\sigma}<\Gamma_{\phi}
$$

where $\Gamma_{\sigma(\phi)}$ is the decay rate of the ungaussiton (inflaton). There are still two cases to be considered, depending on whether the ungaussiton starts oscillations before or after the reheating.

If the ungaussiton starts its oscillations after the inflaton decay, or equivalently, if $m_{\sigma}<\Gamma_{\phi}$, a relevant part of the e-folding number $N$ that depends on $\sigma$ is given by [31]

$$
N\left(t_{f}, t_{*}\right) \supset \frac{1}{24} \sqrt{\frac{\pi}{2}} \alpha^{2} p+\mathcal{O}\left(p^{2}\right),
$$

where $\alpha=2 \sqrt{2 / \pi} \Gamma(5 / 4) \approx 1.45$ is a numerical constant and $p \equiv\left(\bar{\sigma} / M_{P}\right)^{2} \sqrt{m_{\sigma} / \Gamma_{\sigma}}$. The parameter $p$ roughly represents the ratio of the ungaussiton energy density to the total one at the time when the ungaussiton decays. If the ungaussiton dominates the universe before its decay, the resultant curvature perturbations would become highly non-Gaussian, which is inconsistent with the current observations. Hence we require $p \ll 1$. Note that we have assumed that the curvature perturbation dominantly comes from the inflaton, while the ungaussiton gives only small correction to it. This actually gives a severer bound on $p$, i.e., $p \ll O\left(10^{-5}\right)$. Substituting (23) into (19) and (20), we obtain

$$
\begin{aligned}
& \frac{6}{5} f_{\mathrm{NL}}=\frac{\alpha^{6}}{216}\left(\frac{\pi}{2}\right)^{3 / 2} \mathcal{P}_{\zeta} \epsilon^{3}\left(\frac{\Gamma_{\sigma}}{m_{\sigma}}\right)^{-3 / 2}\left\{\left(\frac{\bar{\sigma}}{H_{I} / 2 \pi}\right)^{2}+\log \left(k_{b} L\right)\right\}, \\
& \tau_{\mathrm{NL}}=\frac{\pi^{2} \alpha^{8}}{5184} \mathcal{P}_{\zeta} \epsilon^{4}\left(\frac{\Gamma_{\sigma}}{m_{\sigma}}\right)^{-2}\left\{\left(\frac{\bar{\sigma}}{H_{I} / 2 \pi}\right)^{2}+\log \left(k_{t} L\right)\right\},
\end{aligned}
$$

where $\epsilon$ is one of the slow-roll parameters defined by $\epsilon \equiv M_{P}^{2} / 2\left|V_{\phi} / V\right|^{2}$ ( $V$ is the scalar potential of the inflaton), and it is related to $N_{\phi}$ and $\mathcal{P}_{\zeta}$ as

$$
N_{\phi}=\frac{1}{\sqrt{2 \epsilon} M_{P}}, \quad \mathcal{P}_{\zeta}=\frac{H_{I}^{2}}{8 \pi^{2} \epsilon M_{P}^{2}} .
$$

The WMAP normalization gives $\mathcal{P}_{\zeta} \approx 2 \times 10^{-9}[16]$.

Let us here discuss the magnitudes of the terms in the curly bracket of (24) and (25). Since we are interested in a scalar fluctuating around the origin, the first term should be 
$O(1)$. There are several ways to realize such situation as stated in Sec. I. In order to estimate the magnitude of the second term, we need to specify values of $k$ and $L$. What values of $L$ should be taken depends on the spatial size of the observational data. For instance, suppose that we have observational data on a variable $X$ in a small patch of the sky. Then we should take $L$ to be the size of the patch. We can define a homogeneous part of $X$ by taking the spatial average over the region. The fluctuations is obtained by subtracting the homogeneous mode from $X$. Those fluctuations with the wavelengths larger than $L$ cannot be ditinguished with the homogeneous part, and therefore they are regarded as the homogeneous mode. In our case, since we are interested in the fluctuations of the scales between $k \sim H_{0}$ and $k \sim 10^{4} H_{0}$, we should take $L \sim \mathcal{O}\left(H_{0}^{-1}\right)$, and the second term is $O(1)$ for interested ranges of the scales. Therefore, both terms in the curly brackets are $\mathcal{O}(1)$.

The fact that both terms in the curly brackets are $\mathcal{O}(1)$ means that if the ungaussiton is responsible for the non-Gaussianity of the curvature perturbations, the non-linearity parameters $f_{\mathrm{NL}}$ and $\tau_{\mathrm{NL}}$ have the logarithmic dependence on the spatial scales ${ }^{\mathrm{j}}$. In particular, they increase logarithmically as one goes to small scales.

When does the non-Gaussianity become large? The condition, $f_{\mathrm{NL}}>f_{\mathrm{NL}}^{\min }$, is met if

$$
\epsilon^{3}\left(\frac{\Gamma_{\sigma}}{m_{\sigma}}\right)^{-3 / 2} \gtrsim 7 \times 10^{10}\left(\frac{f_{\mathrm{NL}}^{\min }}{10}\right)
$$

where we have set the terms in the curly brackets on RHS of (24) and (25) to be unity. Equivalenty, we can express the same condition in terms of the Hubble parameter during inflation instead of the slow-roll parameter $\epsilon$;

$$
H_{I} \gtrsim 6 \times 10^{12} \mathrm{GeV}\left(\frac{g_{* d}}{g_{* \mathrm{osc}}}\right)^{1 / 8}\left(\frac{T_{d}}{1 \mathrm{GeV}}\right)^{1 / 2}\left(\frac{T_{\mathrm{osc}}}{10^{8} \mathrm{GeV}}\right)^{-1 / 2}\left(\frac{f_{\mathrm{NL}}^{\min }}{10}\right)^{1 / 6},
$$

where $T_{d}$ and $T_{\text {osc }}$ are the temperatures of the radiation when the ungaussiton decays and when the ungaussiton starts to oscillate, respectively. Here $g_{* d}$ and $g_{* \text { osc }}$ are the effective number of light degrees freedom at $T=T_{d}$ and $T_{\text {osc }}$, respectively.

\footnotetext{
j Note that we have neglected the scale dependence of $\mathcal{P}_{\zeta}$. However, it can be determined by observations, and so, in principle one can extract the scale dependence of the non-linearity parameters.
} 
Let us next consider the other case that the ungaussiton starts its oscillations before the inflaton decay. In this case, we have only to replace $m_{\sigma}$ with $\Gamma_{\phi}$ in Eqs. (27) and (28), employing the sudden decay approximation on the inflaton decay. Hence the condition, $f_{\mathrm{NL}}>f_{\mathrm{NL}}^{\min }$, can be written as

$$
\epsilon^{3}\left(\frac{\Gamma_{\sigma}}{\Gamma_{\phi}}\right)^{-3 / 2} \gtrsim 7 \times 10^{10}\left(\frac{f_{\mathrm{NL}}^{\min }}{10}\right)
$$

or equivalently,

$$
H_{I} \gtrsim 6 \times 10^{12} \mathrm{GeV}\left(\frac{g_{* d}}{g_{* R}}\right)^{1 / 8}\left(\frac{T_{d}}{1 \mathrm{GeV}}\right)^{1 / 2}\left(\frac{T_{R}}{10^{8} \mathrm{GeV}}\right)^{-1 / 2}\left(\frac{f_{\mathrm{NL}}^{\min }}{10}\right)^{1 / 6},
$$

where $T_{R}$ is the reheating temperature of the inflaton, and $g_{* R}$ the effective number of light degree of freedom at $T=T_{R}$.

There is the upper bound on the Hubble parameter during inflation as $H_{I} \lesssim 10^{14} \mathrm{GeV}$ from the WMAP result [16]. Meanwhile, both (28) and (30) give the lower bounds on $H_{I}$. Notice that both the conditions (28) and (30) require the decay temperature be much lower than the reheating temperature, i.e., the life time of the ungaussiton must be rather long. The longevity is necessary for the ungaussiton to give non-negligible contribution to the energy density, and therefore to the non-Gaussianity. It suggests that the ungaussiton must couple only weakly to the visible sector. Suppose that $\sigma$ couples to the visible sector strongly. Then $\sigma$ can be immediately thermalized when the universe is reheated by the inflaton decay, which would significantly suppress the non-Gaussianity. Thus the ungaussiton must be indeed weakly coupled to the visible sector.

In Fig. 1, we show the contours of the non-linearity parameters $f_{\mathrm{NL}}$ and $\tau_{\mathrm{NL}}$ as functions of $T_{d} / T_{\text {osc }}\left(\right.$ or $\left.T_{d} / T_{R}\right)$ and $H_{I}$. In the figure, we set $g_{* \text { osc }}=g_{* d}=g_{* R}=100$ and $\bar{\sigma}=H_{I} / 2 \pi$, and drop the logarithmic dependence for simplicity. We see that the region of large $H_{I}$ and small $T_{d} / T_{\text {osc }}$ (or $T_{d} / T_{R}$ ) is already excluded by the observational constraint, $\left|f_{\mathrm{NL}}\right| \lesssim 100[16]$. We also find that the slopes of the contours do not depend on the nonlinearity parameters: $H_{I} \propto \sqrt{T_{d} / T_{\text {osc }}}$ or $\sqrt{T_{d} / T_{R}}$ for all the non-linearity parameters. This is because both $f_{\mathrm{NL}}$ and $\tau_{\mathrm{NL}}$ depend only on the combination $\epsilon \sqrt{m_{\sigma} / \Gamma_{\sigma}}$ (or $\epsilon \sqrt{\Gamma_{\phi} / \Gamma_{\sigma}}$ ), as can be seen from Eqs. (24) and (25). 


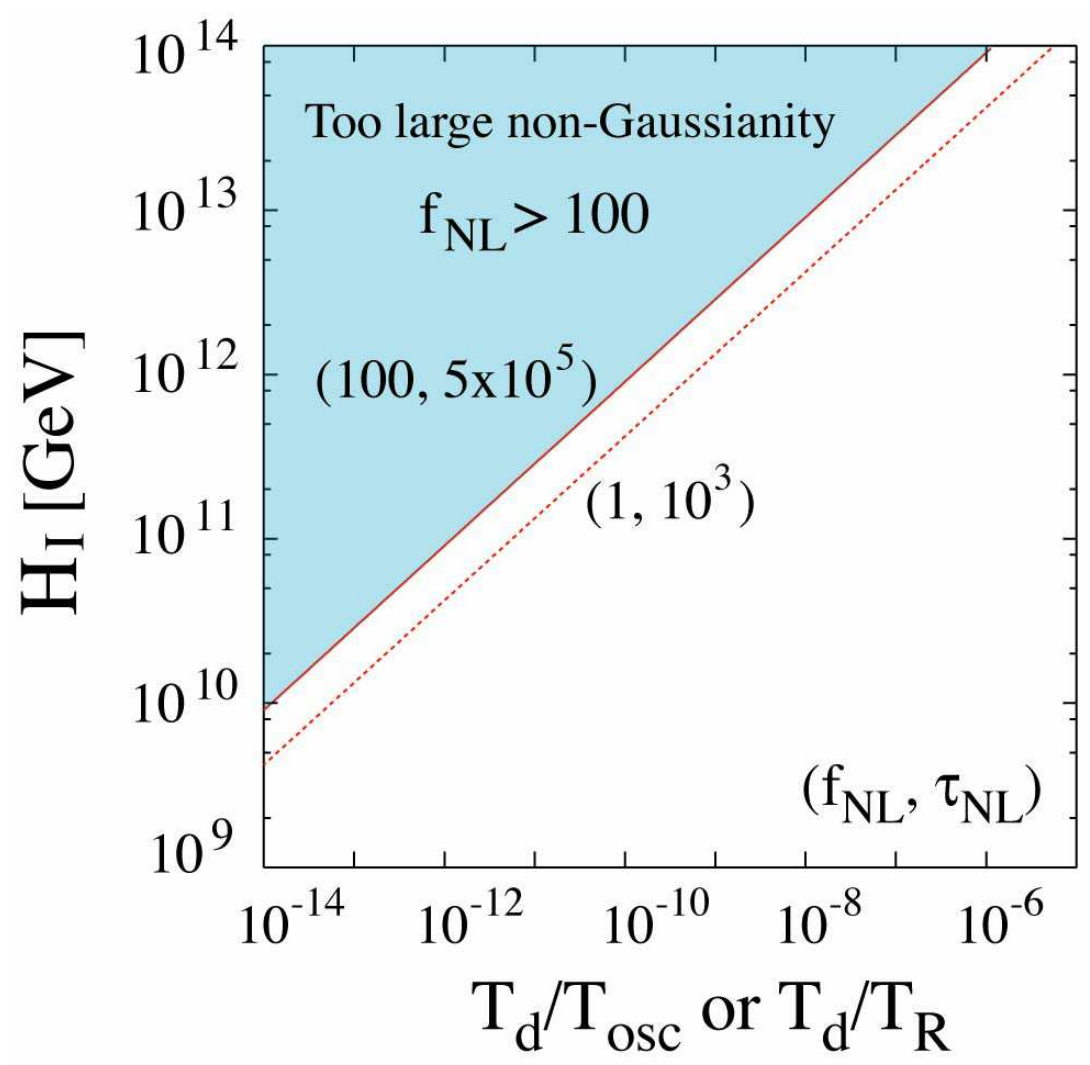

FIG. 1: Contours of the non-linearity parameters $f_{\mathrm{NL}}$ and $\tau_{\mathrm{NL}} ;\left(f_{\mathrm{NL}}, \tau_{\mathrm{NL}}\right)=\left(100,5 \times 10^{5}\right)(\operatorname{solid}$ line) and $\left(1,10^{3}\right)$ (dotted line). Shaded region is already excluded by the WMAP 5yr data. Here we set $g_{* \mathrm{osc}}=g_{* d}=g_{* R}=100$ and $\bar{\sigma}=H_{I} / 2 \pi$, and drop the logarithmic dependence.

The fact that all the non-linearity parameters depend only on one combination of the model parameters means that we have an universal relation between $f_{\mathrm{NL}}$ and $\tau_{\mathrm{NL}}$, which is independent of the model parameters. Such a consistency relation is given by

$$
\begin{aligned}
\tau_{\mathrm{NL}} & =C \mathcal{P}_{\zeta}^{-\frac{1}{3}}\left(\frac{6}{5} f_{\mathrm{NL}}\right)^{\frac{4}{3}}, \\
& \approx 1 \times 10^{3} C f_{\mathrm{NL}}^{4 / 3},
\end{aligned}
$$

where we have used $\mathcal{P}_{\zeta} \simeq 2 \times 10^{-9}[16]$. Here $C$ is a numerical coefficient of order unity 
defined by

$$
C \equiv \frac{\frac{\bar{\sigma}^{2}}{\left(H_{I} / 2 \pi\right)^{2}}+\log k_{t} L}{\left(\frac{\bar{\sigma}^{2}}{\left(H_{I} / 2 \pi\right)^{2}}+\log k_{b} L\right)^{4 / 3}}
$$

As a remarkable fact, when $f_{\mathrm{NL}}>1$, the trispectrum of the curvature perturbations becomes inevitably very large $\tau_{\mathrm{NL}} \gg 1$. Although the current bound on $\tau_{\mathrm{NL}}$ is very weak, we will have much stronger bound in the near future: e.g., $\left|\tau_{\mathrm{NL}}\right| \lesssim 560$ for Planck [32]. Hence if the non-Gaussianity $f_{\mathrm{NL}}>1$ is generated by the ungaussiton mechanism, we should also see the strong non-Gaussianity through the trispectrum, which will be quite useful to distinguish the ungaussiton scenario from the others that predict different consistency relations between the bispectrum and the trispectrum [31, 33, 34].

Let us here show that the consistency relation (31) can be understood in an intuitive way. The bispecturm is roughly given by $\left\langle\zeta^{3}\right\rangle \sim N_{\sigma \sigma}^{3}\left\langle(\delta \sigma)^{6}\right\rangle$, while the trispectrum is $\left\langle\zeta^{4}\right\rangle \sim N_{\sigma \sigma}^{4}\left\langle(\delta \sigma)^{8}\right\rangle$. Therefore, as long as the quadratic term $(\delta \sigma)^{2}$ is the dominant source of the non-Gaussianity, we naively expect $\left\langle\zeta^{4}\right\rangle \sim\left(\left\langle\zeta^{3}\right\rangle\right)^{4 / 3}$, which correctly reproduces $\tau_{\mathrm{NL}} \sim \mathcal{P}_{\zeta}^{-1 / 3} f_{\mathrm{NL}}^{4 / 3} \sim 10^{3} f_{\mathrm{NL}}^{4 / 3}$. We can easily extend this argument to the $n$-th correlator. The leading $n$-th non-linearity parameter defined in a similar fashion, $f_{\mathrm{NL}}^{(n)}$, is roughly equal to $\mathcal{P}_{\zeta}^{(3-n) / 3} f_{\mathrm{NL}}^{n / 3} \sim 10^{3(n-3)} f_{\mathrm{NL}}^{n / 3}$. One can see that this simple argument breaks down if $N_{\sigma}$ is not suppressed as in the curvaton scenario, since the bispectrum would be dominated by $\left\langle\zeta^{3}\right\rangle \sim N_{\sigma}^{2} N_{\sigma \sigma}\left\langle(\delta \sigma)^{4}\right\rangle$. In that case there are different consistency relations [31]. Thus it is very important to test the consistency relations by observations, in order to distinguish different scenarios to produce non-Gaussianity.

As mentioned before, there must be hierarchy between $T_{R}$ (or $T_{\text {osc }}$ ) and $T_{d}$. Since the ungaussiton must decay before the big bang nucleosynthesis (BBN), $T_{d}$ must be higher than $10 \mathrm{MeV}$ [35]. Then, the reheating temperature is bounded below: e.g., $T_{R} \gtrsim 10^{2} \mathrm{GeV}$ for $H_{I}=10^{14} \mathrm{GeV}$, and $T_{R} \gtrsim 10^{9} \mathrm{GeV}$ for $H_{I}=10^{11} \mathrm{GeV}$, in order to have $f_{\mathrm{NL}} \gtrsim 1$. Such high reheating temperature as $T_{R} \gtrsim 10^{9} \mathrm{GeV}$ may lead to the overproduction of the gravitinos [36, 37], when the local SUSY is assumed. One way to ameliorate the tension is to consider the heavy or ultralight gravitino mass: $m_{3 / 2} \gtrsim O(10) \mathrm{TeV}$ or $m_{3 / 2} \lesssim O(10) \mathrm{eV}$. Another is to introduce a late-time entropy production after the ungaussiton decays 38 , 
39]. Note that the density fluctuations after the ungaussiton decays are adiabatic, although they contain sizable non-Gaussianity. Once it becomes adiabatic, the non-Gaussianity will be inherent in the subsequent evolution of the universe, whatever it is like. In particular, the non-Gaussianity does not change even if there is late-time entropy production after the ungaussiton decays, unless isocurvature perturbations exist in the fields that induce entropy production.

Let us here make a comment on the isocurvature perturbations. If the baryon number or the dark matter abundance is fixed before the undaussiton decays, the non-Gaussian isocurvature perturbations are generated. The amplitude of the isocurvature perturbations are smaller than the current bounds, for the non-Gaussianity with $f_{\mathrm{NL}}<100$. Although we do not consider its effects throughout this paper, the isocurvature perturbations, if observed, may provide another clue on the ungaussiton mechanism.

Before closing this section, we note that the symmetries does not have to be exact to realize the ungaussiton scenario. The explicit breaking of the symmetries will generically makes the potential minimum deviate from the origin, and $\bar{\sigma}$ may have a large value. However, if the deviation is the order of $H_{I}$, this is indistinguishable from the non-vanishing $\bar{\sigma}$ due to the fluctuations on large scales beyond the size of the observable universe. Also, as in the case of an axion field with a shift symmetry: $a \rightarrow a+\alpha$, the enhanced-symmetry point may not exist. Even in this case, if the initial position of the scalar happens to be near the minimum, it can be an ungaussiton if it is light during inflation. Therefore our discussions above remain intact in these cases.

\section{MODELS}

Let us here give some models to realize our idea. We will give detailed analysis on each model in the coming paper [40]. One example is the modulus field, which appears in the supergravity/superstring theory and it has a very flat potential. If the position of the modulus field during inflation is deviated from the minimum in the low energy, it leads to the cosmological moduli problem, since the large energy density stored in the modulus field 
can easily modify and spoil the standard evolution of the universe. One of the solutions to the moduli problem is to presume that the moduli fields having symmetry-enhanced points in their field space stay at the points during and after inflation [6, 7, 8, 9, 10], which would greatly suppress the cosmological abundance of the moduli. Such moduli fields become ungaussitons, if they are light during inflation ${ }^{k}$. Since the moduli must decay before BBN starts, the modulus mass must be larger than $O\left(10^{2}\right) \mathrm{TeV}$. Assuming that the modulus has couplings with the standard-model particles suppressed by the Planck scale, the decay temperature is given by around $O(100) \mathrm{MeV}$ for the modulus mass of $10^{3} \mathrm{TeV}$. From Fig. 1, one can see that large non-Gaussianity is generated for e.g. $H_{I}=10^{11} \mathrm{GeV}$ and $T_{R}=10^{10} \mathrm{GeV}$. In the case of the moduli, the needed hierarchy between $T_{R}$ and $T_{d}$ is realized due to the weakness of the modulus couplings.

Another is a right-handed sneutrino, $\tilde{N}$, a superpartner of the right-handed neutrino, $N$. The right-handed sneutrino is odd under the $R$-parity, which determines the origin of $\tilde{N}$. If the mass of $\tilde{N}$ is light during inflation, it can be a ungaussiton. If the neutrino Yukawa coupling $y$ is small enough and if the mass of $\tilde{N}$ is relatively light, the decay of $\tilde{N}$ can be delayed. Also one can neglect the thermal production of $\tilde{N}$ for sufficiently small Yukawa couplings. Therefore, it is relatively easy to satisfy the relation (30) if the model is close to the case of the Dirac neutrino. For instance, the decay temperature is roughly estimated by

$$
T_{d} \sim 0.1 y \sqrt{m_{\tilde{N}} M_{P}}
$$

where $m_{\tilde{N}}$ is the mass of $\tilde{N}$. Therefore, large non-Gaussianity is generated for e.g. $y=10^{-6}, m_{\tilde{N}}=1 \mathrm{TeV}, H_{I}=10^{14} \mathrm{GeV}$ and $T_{R}=10^{7} \mathrm{GeV}$. Note that the gravitationally produced right-handed sneutrino cannot explain the baryon asymmetry through leptogenesis [42], since the baryon asymmetry would have too large isocurvature perturbations.

The last example is a flat direction of the supersymmetric standard model (SSM). There are many flat directions in SSM, and they are parametrized by a gauge-invariant monomial such as $u d d$ and $e L L$. The flat directions are composed of squarks, sleptons and

k The abundance of the such modulus field was investigated in Ref. 41] to study how the moduli problem is relaxed by the mechanism. 
Higgs, and therefore, the origins of the flat directions are determined by the SM gauge symmetry. In addition, we expect that some of the flat directions may be light during inflation, since there are many. However, since they interact with the standard-model particle through the SM gauge interactions, they might be easily thermalized after the reheating. Interestingly, there is one possibility: $Q$-balls [43]. After the flat direction starts oscillating, it generically experiences spatial instabilities and deforms into non-topological solitons called $Q$-balls ${ }^{1}$. Once the $Q$-balls are formed, their life time is typically very long since the decay and evaporation processes occur only around the surfaces of the $Q$ balls [44]. The relation (30) can be satisfied for certain values of the inflation scale and the reheating temperature. For instance, we take $H_{I}=10^{14} \mathrm{GeV}$. Assuming the gravity mediation or the anomaly-mediation [45], the typical charge of the $Q$-ball (of the gravitymediaiton type) is $Q \sim 10^{19}$ [46]. Such $Q$-balls decay or evaporate depending on the details of the flat directions, but the typical decay or evaporation temperature is between $\mathcal{O}(1) \mathrm{GeV}$ to $\mathcal{O}(100) \mathrm{GeV}$. Then the relation (30) is met for $T_{R} \simeq 10^{7} \sim 10^{9} \mathrm{GeV}$. What makes this scenario slightly complicated is that the decay temperature is related to the charge of the $Q$-balls, which in turn depends on the initial amplitude. Therefore, in this model using the $Q$-balls, we also have to take account of the non-Gaussianity produced by the so-called modulated reheating scenario. Although we expect that the results derived in the previous section can be qualitatively valid even in this case, there must be quantitative difference. The detailed analysis of this scenario is beyond the scope of this paper, and we leave it for the future work. Nevertheless we would like to emphasize that the flat directions in SSM can be ungaussitons, which makes us to believe that the ungaussiton mechanism is indeed feasible and that one can find further explicit models.

Each scenario described above has its own implications on the inflationary scale, the mass scale and the lifetime of the ungaussiton. We will discuss this issue in a separate paper.

\footnotetext{
${ }^{1}$ Note that the spatial scale of the $Q$-balls are much smaller than the cosmological scales of our interest.
} 


\section{DISCUSSION AND CONCLUSIONS}

We would like to emphasize here that the ungaussiton is different from a curvaton [47], which has been extensively studied as a possible source for the non-Gaussianity [17, 48, 49, 50, 51, 52, 53, 54]. The original motivation of the curvaton scenario was to explain the adiabatic density fluctuations by the fluctuations in the curvaton field. Therefore it is crucial for the curvaton to develop a large non-zero expectation value during inflation. The large expectation value helps the curvaton to dominate the energy density of the universe, or at least it makes the energy density of the curvaton sizable if it does not dominate. At the same time, the fluctuations in the energy density of the curvaton field becomes almost Gaussian with relatively small non-Gaussianity. On the other hand, the ungaussiton has a classically vanishing field value during inflation. Therefore its energy density exhibits strong non-Gaussianity, and it is not responsible for the almost Gaussian curvature perturbations observed in the CMB. Note that this difference results in the smaller number of the parameters in the ungaussiton scenario relative to the curvaton scenario, which makes the former more predictive. This is because the amplitude of the ungaussiton is determined solely by the Hubble parameter during inflation, while the amplitude of the curvaton is a free parameter in the curvaton scenario $\mathrm{m}$. Thanks to this feature, we have obtained the consistency relation between the bispectrum and the trispectrum (31). Although the techniques to calculate the non-Gaussianity are common to the case of the curvaton, the background philosophy is different: the presence of the symmetry-enhanced points in the field space is the key idea for our arguments.

Lastly, let us discuss how the ungaussiton can remain around the minimum of the potential during inflation. Since the mass is assumed to be lighter than the Hubble parameter, one may wonder why the ungaussiton can find its origin and sit there during and after inflation. Our answer is as follows.

\footnotetext{
${ }^{m}$ In the ungaussiton scenario, there is numerical uncertainty of order unity in the effective amplitude of $\sigma$, since it arises from the purely quantum fluctuations. Still, we have less number of the dimensionful parameters.
} 
There are several ways to infer how the initial position is determined, although we cannot decisively predict it unless the dynamics before (or during) inflation is unravelled. If the position is determined in a probabilistic way, the probability distribution must have its extremum at the origin, if the theory respects the symmetry around the origin. (This probability distribution has nothing to do with the quantum fluctuations. The initial condition we are talking here is about the position before the ungaussiton starts to fluctuate.) We do not know if the extremum is either (local) minimum or maximum. If the probability becomes maximum at the origin, the scalar field likely sits at the origin at the beginning of the universe. Such scalar can be an ungaussiton. On the other hand, the symmetry would be spontaneously broken if the probability is minimum at the origin, which is more suitable for the curvaton scenario.

One can also imagine that the initial position is determined by some dynamics. For instance, if there is another inflationary phase before the primordial inflation, the scalar field may acquire a positive mass of the order of the Hubble parameter around the origin. Then the scalar will settle down at the origin during the pre-inflation. Or, there might be a radiation-dominated phase after the pre-inflation and before the last inflation starts. If the ungaussiton is thermalized during this phase, it will stay near the origin when the last inflation starts.

If the initial position is somehow set around the origin as described above, the ungaussiton will start to fluctuate about the origin during the last inflation. If it lasted for 50-60 e-foldings, it still remains around the origin. However, if the last inflation lasted for much longer period, the situation changes.

As the inflation lasts longer, the fluctuations will be accumulated, and the initial condition set before the inflation becomes less important, and finally, it will be forgotten in the end. The asymptotic distribution is known as the $\mathrm{BD}$ distribution. If it is reached, the ungaussiton takes a value of $\mathcal{O}\left(H_{I}^{2} / m\right)$ in most regions of the whole universe. Our observable universe may happen to be in a (small) region where the ungaussiton takes a value of $O\left(H_{I}\right)$. This is a fine-tuning if $m \ll H_{I}$, since such regions are quite rare [12]. However, this is not the case if the effective mass $m$ is not much smaller than $H_{I}$. If we 
take $m=0.1 H_{I}$, for instance, the RMS of the field values is $\mathcal{O}\left(10 H_{I}\right)$. Therefore, we can find a (sub)universe where the spatial average of the ungaussiton field is $O\left(H_{I}\right)$ without severe fine-tunings. Note that the fluctuations of the ungaussiton in the observable universe are not damped significantly, for $m \lesssim 0.1 H_{I}$, because the suppression factor is given by $\sim \exp \left(-\frac{m^{2}}{H_{I}^{2}} N_{*}\right)$ where $N_{*}$ is the e-folding number from the time when the mode crossed the horizon to the time of the inflation end. Such possibility can be naturally realized in the context of supergravity. In supergravity, a scalar field generically acquires a mass of $O\left(H_{I}\right)$ during inflation. Depending on a precise form of the Kahler potential, the effective mass can be lighter than $H_{I}$. (One can even make the correction vanish if the Kahler potential is finely tuned.) Therefore, without severe fine-tunings, it is possible that the effective mass is only slightly smaller than $H_{I}$.

After all, we cannot tell the initial position of a scalar field due to our ignorance regarding the scalar dynamics in the inflationary phase. However, without any theoretical prejudice, we can still expect that a scalar field possibly sits at the origin, simply because the origin is so special in its field space.

If there are the symmetry-enhanced points, we believe it plausible that the scalar fields stay at the points during and after inflation. If some of them are light during inflation, they acquire quantum fluctuations. Such light scalars are ungaussitons: their energy densities are necessarily highly non-Gaussian! Importantly, such non-Gaussianity must have been imprinted in the CMB spectrum. For the effects to be large enough to be actually measured by the ongoing and planned observations, the relations Eqs. (28) or (30) must be satisfied. Note that the criterion for a scalar field to be an ungaussiton is not strict at all. Any scalar field charged under some symmetries can become an ungaussiton, if it is light during inflation. If such scalars are mediocre in nature, we may well expect that there are indeed such ungaussitons satisfying the relations Eq. (28) or (30).

We do not claim that the ungaussiton mechanism is superior to the curvaton scenario. These two are independent and interesting possibilities to generate sizable non-Gaussianity in the CMB spectrum, and as we have mentioned before, the ungaussiton scenario predicts distinctive bispectrum and trispectrum: the enhancement at the small scales and the 
consistency relation (31). Therefore, the future observations may be able to tell one from the other.

Acknowledgments: F.T. would like to thank Matt Buckley and Sourav Mandal for suggesting the name of the particle. We would also like to thank M. Kawasaki for comments. This work was supported by World Premier International Research Center Initiative (WPI Program), MEXT, Japan.

[1] See for example, J. Polchinski, String Theory, Cambridge Monographs on Mathematical Physics, 1998.

[2] G. D. Coughlan et al Phys. Lett. B 131 (1983) 59; T. Banks, D. B. Kaplan and A. E. Nelson, Phys. Rev. D 49 (1994) 779; B. de Carlos et al Phys. Lett. B 318 (1993) 447.

[3] M. Endo, K. Hamaguchi and F. Takahashi, Phys. Rev. Lett. 96, 211301 (2006);

S. Nakamura and M. Yamaguchi, Phys. Lett. B 638, 389 (2006).

[4] M. Dine, R. Kitano, A. Morisse and Y. Shirman, Phys. Rev. D 73, 123518 (2006).

[5] M. Endo, K. Hamaguchi and F. Takahashi, Phys. Rev. D 74, 023531 (2006).

[6] M. Dine, Y. Nir and Y. Shadmi, Phys. Lett. B 438, 61 (1998).

[7] L. Kofman, A. Linde, X. Liu, A. Maloney, L. McAllister and E. Silverstein, JHEP 0405, 030 (2004) arXiv:hep-th/0403001.

[8] S. Watson, Phys. Rev. D 70, 066005 (2004) arXiv:hep-th/0404177.

[9] S. Cremonini and S. Watson, Phys. Rev. D 73, 086007 (2006) arXiv:hep-th/0601082.

[10] B. Greene, S. Judes, J. Levin, S. Watson and A. Weltman, JHEP 0707, 060 (2007) arXiv:hep-th/0702220.

[11] Y. B. Zeldovich and A. A. Starobinsky, Sov. Phys. JETP 34, 1159 (1972) [Zh. Eksp. Teor. Fiz. 61, 2161 (1971)]; L. P. Grishchuk, Lett. Nuovo Cim. 12, 60 (1975) [Erratum-ibid. 12, 432 (1975)]; S. G. Mamaev, V. M. Mostepanenko and A. A. Starobinsky, Zh. Eksp. Teor. Fiz. 70 (1976) 1577; A. A. Grib, S. G. Mamaev and V. M. Mostepanenko, Gen. Rel. Grav. 7 (1976) 535 . 
[12] A. D. Linde and V. F. Mukhanov, Phys. Rev. D 56, 535 (1997) arXiv:astro-ph/9610219;

A. Linde and V. Mukhanov, JCAP 0604, 009 (2006) arXiv:astro-ph/0511736.

[13] R. Durrer, M. Gasperini, M. Sakellariadou and G. Veneziano, Phys. Rev. D 59, 043511 (1999) arXiv:gr-qc/9804076; Phys. Lett. B 436, 66 (1998) arXiv:astro-ph/9806015; M. Gasperini and G. Veneziano, Phys. Rev. D 59, 043503 (1999) arXiv:hep-ph/9806327.

[14] T. S. Bunch and P. C. W. Davies, Proc. Roy. Soc. Lond. A 360, 117 (1978).

[15] A. P. S. Yadav and B. D. Wandelt, arXiv:0712.1148 [astro-ph].

[16] E. Komatsu et al. [WMAP Collaboration], arXiv:0803.0547 [astro-ph].

[17] D. H. Lyth, JCAP 0606, 015 (2006) arXiv:astro-ph/0602285.

[18] M. Kawasaki, K. Nakayama, T. Sekiguchi, T. Suyama and F. Takahashi, arXiv:0808.0009 [astro-ph].

[19] A. A. Starobinsky, JETP Lett. 42 (1985) 152 [Pisma Zh. Eksp. Teor. Fiz. 42 (1985) 124].

[20] M. Sasaki and E. D. Stewart, Prog. Theor. Phys. 95, 71 (1996).

[21] M. Sasaki and T. Tanaka, Prog. Theor. Phys. 99, 763 (1998) arXiv:gr-qc/9801017.

[22] D. H. Lyth, K. A. Malik and M. Sasaki, JCAP 0505, 004 (2005) arXiv:astro-ph/0411220.

[23] J. M. Maldacena, JHEP 0305, 013 (2003) arXiv:astro-ph/0210603.

[24] D. Seery and J. E. Lidsey, JCAP 0509, 011 (2005) arXiv:astro-ph/0506056.

[25] D. Seery, J. E. Lidsey and M. S. Sloth, JCAP 0701, 027 (2007) arXiv:astro-ph/0610210.

[26] F. Arroja and K. Koyama, arXiv:0802.1167 [hep-th].

[27] C. T. Byrnes, M. Sasaki and D. Wands, Phys. Rev. D 74, 123519 (2006) arXiv:astro-ph/0611075].

[28] D. H. Lyth and Y. Rodriguez, Phys. Rev. Lett. 95, 121302 (2005) arXiv:astro-ph/0504045.

[29] L. Boubekeur and D. H. Lyth, Phys. Rev. D 73, 021301 (2006) arXiv:astro-ph/0504046.

[30] D. H. Lyth, JCAP 0712, 016 (2007) [arXiv:0707.0361 [astro-ph]].

[31] K. Ichikawa, T. Suyama, T. Takahashi and M. Yamaguchi, arXiv:0802.4138 [astro-ph].

[32] N. Kogo and E. Komatsu, Phys. Rev. D 73, 083007 (2006) arXiv:astro-ph/0602099.

[33] T. Suyama and M. Yamaguchi, Phys. Rev. D 77, 023505 (2008) arXiv:0709.2545 [astro-ph]].

[34] E. I. Buchbinder, J. Khoury and B. A. Ovrut, arXiv:0710.5172 [hep-th]. 
[35] M. Kawasaki, K. Kohri and N. Sugiyama, Phys. Rev. Lett. 82, 4168 (1999) arXiv:astro-ph/9811437]; Phys. Rev. D 62, 023506 (2000) arXiv:astro-ph/0002127; S. Hannestad, Phys. Rev. D 70, 043506 (2004) arXiv:astro-ph/0403291; K. Ichikawa, M. Kawasaki and F. Takahashi, Phys. Rev. D 72, 043522 (2005) arXiv:astro-ph/0505395.

[36] S. Weinberg, Phys. Rev. Lett. 48, 1303 (1982).

[37] L. M. Krauss, Nucl. Phys. B 227, 556 (1983).

[38] D. H. Lyth and E. D. Stewart, Phys. Rev. D 53, 1784 (1996). arXiv:hep-ph/9510204.

[39] M. Kawasaki and F. Takahashi, Phys. Lett. B 618, 1 (2005) arXiv:hep-ph/0410158.

[40] T. Suyama and F. Takahashi, in preparation.

[41] G. N. Felder, L. Kofman and A. D. Linde, JHEP 0002, 027 (2000).

[42] M. Fukugita and T. Yanagida, Phys. Lett. B 174, 45 (1986).

[43] S. R. Coleman, Nucl. Phys. B 262, 263 (1985) [Erratum-ibid. B 269, 744 (1986)].

[44] A. G. Cohen, S. R. Coleman, H. Georgi and A. Manohar, Nucl. Phys. B 272, 301 (1986).

[45] L. Randall and R. Sundrum, Nucl. Phys. B 557, 79 (1999);

G. F. Giudice, M. A. Luty, H. Murayama and R. Rattazzi, JHEP 9812, 027 (1998);

J. A. Bagger, T. Moroi and E. Poppitz, JHEP 0004, 009 (2000).

[46] S. Kasuya and M. Kawasaki, Phys. Rev. D 62, 023512 (2000).

[47] K. Enqvist and M. S. Sloth, Nucl. Phys. B 626, 395 (2002);

D. H. Lyth and D. Wands, Phys. Lett. B 524, 5 (2002);

T. Moroi and T. Takahashi, Phys. Lett. B 522, 215 (2001) [Erratum-ibid. B 539, 303 (2002)].

[48] D. H. Lyth, C. Ungarelli and D. Wands, Phys. Rev. D 67, 023503 (2003) arXiv:astro-ph/0208055].

[49] N. Bartolo, S. Matarrese and A. Riotto, Phys. Rev. D 69, 043503 (2004) arXiv:hep-ph/0309033.

[50] N. Bartolo, E. Komatsu, S. Matarrese and A. Riotto, Phys. Rept. 402, 103 (2004) arXiv:astro-ph/0406398.

[51] K. Enqvist and S. Nurmi, JCAP 0510, 013 (2005) arXiv:astro-ph/0508573. 
[52] K. A. Malik and D. H. Lyth, JCAP 0609, 008 (2006) arXiv:astro-ph/0604387.

[53] M. Sasaki, J. Valiviita and D. Wands, Phys. Rev. D 74, 103003 (2006) arXiv:astro-ph/0607627.

[54] Q. G. Huang, arXiv:0801.0467 [hep-th]. 